\title{
Does wearing a non-medical face mask cause changes in cerebral hemodynamics?
}

Fischer, Jonas, Kobayashi Frisk, Lisa, Tachtsidis, Ilias, Delgado Mederos, Raquel, Mayos Pèrez, Mercedes, et al.

Jonas B. Fischer, Lisa Kobayashi Frisk, llias Tachtsidis, Raquel Delgado Mederos, Mercedes Mayos Pèrez, Felix Scholkmann, Turgut Durduran, "Does wearing a non-medical face mask cause changes in cerebral hemodynamics?," Proc. SPIE 11920, Diffuse Optical Spectroscopy and Imaging VIII, 119200J (9 December 2021); doi: 10.1117/12.2615227 


\title{
Does wearing a non-medical face mask cause changes in cerebral hemodynamics?
}

\author{
Jonas B. Fischer, ${ }^{1, *}$ Lisa Kobayashi Frisk, ${ }^{1}$ Ilias Tachtsidis, ${ }^{2}$ \\ Raquel Delgado Mederos, ${ }^{3}$ Mercedes Mayos Pèrez, ${ }^{4,5}$ Felix Scholkmann, ${ }^{6}$ and \\ Turgut Durduran ${ }^{1,7}$ \\ ${ }^{1}$ ICFO-Institut de Ciències Fotòniques, The Barcelona Institute of Science and Technology, Castelldefels \\ (Barcelona), Spain; ${ }^{2}$ Department of Medical Physics and Biomedical Engineering, University College London, \\ London, London, UK; ${ }^{3}$ Hospital de la Santa Creu i Sant Pau, Department of Neurology, Sant Pau Biomedical \\ Research Institute, Barcelona, Spain; ${ }^{4}$ Hospital de la Santa Creu i Sant Pau, Department of Respiratory \\ Medicine, Sleep Unit, Barcelona, Spain, ${ }^{5}$ CIBER Enfermedades Respiratorias (CibeRes) (CB06/06), Madrid, \\ Spain; ${ }^{6}$ University Hospital Zurich, University of Zurich, Department of Neonatology, Biomedical Optics \\ Research Laboratory, Zurich, Switzerland; ${ }^{7}$ Institució Catalana de Recerca i Estudis Avançats (ICREA), \\ Barcelona, Spain \\ *jonas.fischer@icfo.eu
}

\begin{abstract}
We present a study investigating the effect of non-medical face masks (FFP2 and surgical) on cerebral hemodynamics measured by transcranial hybrid diffuse optics, and on systemic physiology in 13 healthy adults (age: 23-33 years). @ 2021 The Author(s)
\end{abstract}

\section{Introduction}

The COVID-19 pandemic has led many countries to introduce regulations obliging or recommending the public to wear facial masks [1] as a preventative method to minimize the spread of the disease.

Discussions are ongoing about possible adverse effects of mask wearing (e.g. dizziness, headaches, exhaustion, fainting), especially in elderly people and those wearing the mask for longer times and during physical activity. Currently, research about those possible adverse effects is currently being carried out. For example, Chan et al. [2] found in a recent study in elderly (age: $76.4 \pm 6.1$ years) no statistically significant change in arterial oxygenation $\left(\mathrm{SpO}_{2}\right)$ after wearing a mask for one hour. Recently, Law et al. [3] published the first result of a fMRI study (eight subject, age: $44 \pm 15$ years) showing that wearing a mask increased the BOLD signal by $30 \%$ on average, increases end-tidal carbon dioxide pressure $\left(\mathrm{EtCO}_{2}\right)$, but does not alter significantly task-induced changes in the BOLD signal. Clearly more research is needed.

To this end, we have studied the effect of wearing non-medical face masks (FFP2 and surgical) on cerebral hemodynamics, oxygenantion and metabolism as well as systemic physiology in a group of young healthy volunteers using transcranical, hybrid, near-infrared diffuse optical spectroscopy. This is the first study that uses optical neuroimaging to study possible effects of mask-wearing on cerebral hemodynamic and systemic physiological parameters.

\section{Methods}

The study protocol was approved by the ethical committee of Hospital Clinic Barcelona (Spain) and all participants signed informed consent. Young adults between 20 and 35 years of age were recruited from the general population of a research institute adhering to COVID-19 regulations. We have ruled out existing cardiovascular, neurological or respiratory conditions assessed by a questionnaire.

A ten minute baseline data was acquired without the use of a mask while the subjects were asked to sit calmly in front of a computer and read a scientific paper of interest. After the baseline the mask was put on and data was acquired for another ten minutes. All subjects were measured on two separate days - FFP2 mask was utilized on day one, and a surgical mask was utilized on day two.

Data was acquired bilaterally and continuously using optical probes placed over the frontal lobes while avoiding the sinuses. The probes utilized transcranial diffuse correlation spectroscopy (DCS) and time-resolved nearinfrared spectroscopy (TR-NIRS) to measure microvascular cerebral blood flow (CBF), cerebral blood oxygen saturation $\left(\mathrm{StO}_{2}\right)$, and total hemoglobin concentration (THC) [4]. Various systemic physiological signals such as arterial blood pressure (ABP), heart rate (HR), $\mathrm{SpO}_{2}$ (all with Finapres, Finapres Medical Systems BV, Netherlands), respiratory rate (RR), $\mathrm{EtCO}_{2}$ (all by Capnostream 20p, Medtronic, Minneapolis, USA) and transcutaneous carbon dioxide partial pressure $\left(\mathrm{TCO}_{2}\right)$ (SenTec Digital monitor, SenTec AG, Switzerland) were monitored were also continuously acquired. Since, in the case of mask wearing, the nasal cannula used to measure $\mathrm{EtCO}_{2}$ may 
be influenced by the carbon dioxide $\left(\mathrm{CO}_{2}\right)$ trapped between face and mask, its readings are not reliable in this scenario, therefore, we opted for the $\mathrm{TCO}_{2}$ measurement.

In order to test the effect of mask wearing, the data has been normalized to the last five minutes of the baseline period and compared to the last seven minutes while the mask was worn. All parameters were smoothed with the rloess method using a 30 second window to reduce physiological noise. Changes in hemodynamic parameters of both hemispheres were averaged. Since the same subject was measured twice, linear mixed effect models were utilized for statistical analysis. We have tested whether each mask-type is statistically different from zero and whether the difference between the mask-types is statistically different considering the "false discovery rate" (FDR) correction for the p-value and a significance level of 0.05 .

\section{Results}

Thirteen volunteers (median age 27.0 years [range, 23 to 33 years], 6 female [46\%]) were recruited. Figure 1 shows time traces for the population average of the changes in $\mathrm{CBF}$ (panel A), $\mathrm{StO}_{2}$ (panel B), $\mathrm{HR}$ (panel C) and $\mathrm{TCO}_{2}$ (panel D) for both mask-types.

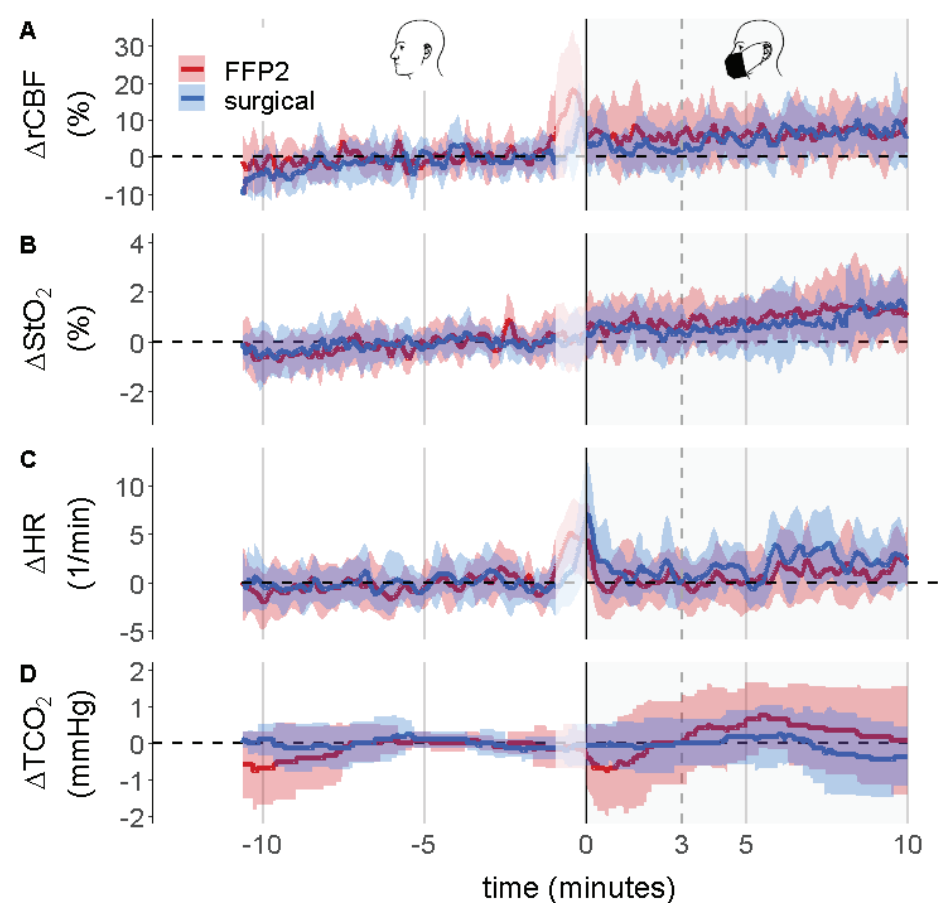

Fig. 1. Time traces of $\mathrm{CBF}(\mathrm{A}), \mathrm{StO}_{2}(\mathrm{~B}), \mathrm{HR}(\mathrm{C})$ and $\mathrm{TCO}_{2}$ (D) shown as the population average for both mask types (FFP2 in red, surgical in blue). The shaded area indicates the standard deviation.

Small but significant changes in $\mathrm{CBF}$ and $\mathrm{StO}_{2}$ were detected for both mask-types. $\mathrm{CBF}$ increased by 6.5 (95\% confidence interval (CI): $2.6,10) \%$ for the FFP2 mask and by $6.2(95 \%$ CI: $2.4,9.9) \%$ for the surgical mask. $\mathrm{StO}_{2}$ increased by 0.9 (95\% CI: $0.2,1.7) \%$ for the FFP2 mask and by 0.9 (95\% CI: $0.1,1.6) \%$ for the surgical mask. THC only increased significantly for the FFP2 mask by 0.9 (95\% CI: $0.3,1.5) \mu \mathrm{M}$. All hemodynamic parameters did not differ between the two mask-types.

As further observed, the systemic parameters such as $\mathrm{TCO}_{2}$ and $\mathrm{SpO}_{2}$ did not significantly change due to wearing a mask, while mean arterial blood pressure (MAP) and HR increased significantly for the surgical mask by 4.1 ( $95 \%$ CI: $0.5,7.6) \mathrm{mmHg}$ and 2.0 (95\% CI: $1.0,3.1)$ beats per minute, respectively. RR decreased significantly for the FFP2 mask by $3.2(95 \% 5.4,1.1)$ breaths per minute. In the systemic physiology only a significant difference between both mask-types was detected for HR, the difference was 1.2 (95\% CI: 0.0, 2.4) beats per minute.

A detailed overview of all results will be presented.

\section{Discussion}

Our findings show that wearing a face mask leads to statistically significant changes in the cerebral hemodynamics $\left(\mathrm{CBF}\right.$ and $\left.\mathrm{StO}_{2}\right)$. The changes are minimal and comparable to the values observed in typical neuroimaging studies for standard tasks such as basic cognitive, visual or motor tasks [5-7]. They are representative of the lower limit of changes observed in daily life. Here, we did not account for potential confounding effects due to discomfort of 
probes, removal/ placement of masks and the order of studies, which may be a cause of increased MAP and HR in for the surgical mask. Generally, these stressors are part of daily life and the addition of mask use did not alter the physiology at a level of potential concern.

Compared to the results of Law et al. [3] we have also observed an increase in $\mathrm{EtCO}_{2}$ while wearing a mask, but we discarded the parameter after evaluation as being unreliable due to the re-breathing of $\mathrm{CO}_{2}$, instead we trusted the $\mathrm{TCO}_{2}$ reading, which did not show a change. Generally, the observed increase of 30\% in the BOLD signals compared to our detected increase in $\mathrm{CBF}$ and $\mathrm{StO}_{2}$ does indeed suggest that mask wearing has an effect on cerebral hemodynmics, although it may not necessarily be a $\mathrm{CO}_{2}$ effect, since, as already mentioned $\mathrm{TCO}_{2}$ did not change.

Our conclusion is that while the changes were measurable, they do not suggest any reason for concern for mask use during daily life activities in healthy young adults at rest. Subjects with pre-existing respiratory problems, undergoing physical activity, affected by high altitudes or elderly may behave differently and further research is needed to investigate possible adverse effects. Our technologies can be utilized for these kind of research questions.

Another important conclusion is that neuroimaging studies using cerebral hemodynamics as a surrogate for neuronal function should carefully evaluate mask use as potential confounder. Our advice would be to take alternate precautions to prevent risks between the subjects and operators. The Society of Neuroscience in Anesthesiology and Critical Care has recently released their recommendations for neuroscience research during the COVID-19 pandemic and stressed the use of personal protective equipment [8]. Our study provides additional data for such recommendations.

Finally, since both DCS and TR-NIRS are suitable to use during realistic tasks, e.g. cognitive or motor, they could be utilized to study whether the measured clinically insignificant changes could alter important aspects of professional life such as attention, reaction times and more.

\section{Acknowledgments \& disclosures}

This work leading to the results was funded by la Fundació La Marató de TV3 (201709.31, 201724.31); Fundació CELLEX Barcelona; Agencia Estatal de Investigación (PHOTOMETABO, PID2019-106481RBC31/10.13039/501100011033, PRE2018-085082); the "Severo Ochoa" (CEX2019-000910-S); "laCaixa" (LlumMedBcn); Institució CERCA, AGAUR-Generalitat (2017 SGR 1380); RIS3CAT (CECH, 001-P-001682); LASERLAB-EUROPE V and EU Horizon 2020 (BitMap 675332, VASCOVID 101016087).

We disclose the following: ICFO has equity ownership in the spin-off company HemoPhotonics S.L. Potential financial conflicts of interest and objectivity of research have been monitored by ICFO's Knowledge \& Technology Transfer Department. No financial conflicts of interest were identified.

\section{References}

1. D. K. Chu, E. A. Akl, S. Duda, K. Solo, S. Yaacoub, H. J. Schünemann, A. El-harakeh, A. Bognanni, T. Lotfi, M. Loeb et al., "Physical distancing, face masks, and eye protection to prevent person-to-person transmission of sars-cov-2 and covid-19: a systematic review and meta-analysis," The Lancet (2020).

2. N. C. Chan, K. Li, and J. Hirsh, "Peripheral oxygen saturation in older persons wearing nonmedical face masks in community settings," JAMA 324, 2323-2324 (2020).

3. C. S. Law, P. S. Lan, and G. H. Glover, "Effect of wearing a face mask on fmri bold contrast," NeuroImage p. 117752 (2021).

4. T. Durduran, R. Choe, W. B. Baker, and A. G. Yodh, "Diffuse optics for tissue monitoring and tomography," Rep Prog Phys 73, 076701 (2010).

5. V. Quaresima, M. Ferrari, A. Torricelli, L. Spinelli, A. Pifferi, and R. Cubeddu, "Bilateral prefrontal cortex oxygenation responses to a verbal fluency task: a multichannel time-resolved near-infrared topography study," J. biomedical optics 10, 011012 (2005).

6. T. Durduran, G. Yu, M. G. Burnett, J. A. Detre, J. H. Greenberg, J. Wang, C. Zhou, and A. G. Yodh, "Diffuse optical measurement of blood flow, blood oxygenation, and metabolism in a human brain during sensorimotor cortex activation," Opt. letters 29, 1766-1768 (2004).

7. K. Uludağ, D. J. Dubowitz, E. J. Yoder, K. Restom, T. T. Liu, and R. B. Buxton, “Coupling of cerebral blood flow and oxygen consumption during physiological activation and deactivation measured with fmri," Neuroimage 23, 148-155 (2004).

8. P. E. Vlisides, K. M. Vogt, D. Pal, E. Schnell, W. M. Armstead, A. M. Brambrink, P. Kuo, P. Nelson, S. Vacas, N. Goettel et al., "Roadmap for conducting neuroscience research in the covid-19 era and beyond: Recommendations from the snacc research committee," J. Neurosurg. Anesthesiol. (2021). 